\title{
Correlation between liver signal intensity in hepatobiliary phase of Gd-EOB-DTPA enhancement and liver function reserves
}

Natthaporn Tanpowpong, Teerasak Phewplung

Department of Radiology, Faculty of Medicine, Chulalongkorn University, Bangkok 10330, Thailand

\begin{abstract}
Background: Gadolinium diethylenetriamine-pentaacetic acid (Gd-EOB-DTPA) is a developed agent with preferential uptake by hepatocytes. A rapid and specific hepatocyte uptake with biliary excretion was observed of approximately $50 \%$ of the injected dose. The amount of contrast uptake is thought to be related to reserve liver function.

Objectives: To evaluate correlation between liver signal intensity in the hepatobiliary phase of Gd-EOB-DTPA and reserved liver function by using the model score for end-stage liver disease (MELD).

Methods: All patients who underwent magnetic resonance (MR) imaging with Gd-EOB-DTPA were retrospectively collected. The patients with serum creatinine level higher than $1.5 \mathrm{mg} / \mathrm{dL}$ or patients without available data to estimate MELD score were excluded. Thirty-six patients were enrolled. A signal-to-noise ratio (SNR) in the liver parenchyma on a fat-suppressed three dimensional fast spoiled-gradient recalled echo sequence images before and 20 minutes after contrast injection were measured and calculated on PACS by two radiologists. The MELD score was determined and interobserver reliability was estimated.

Results: Among 36 patients, we found a negative relationship between the percentage enhancement and the MELD score $(P<0.01, r=0.545)$. The SNR at 20 minutes after Gd-EOB-DTPA injection also had a negative relationship with the MELD score with statistical significance $(P<0.01, r=0.460)$. Interobserver reliability was 0.675 .

Conclusion: The percentage enhancement in hepatobiliary phase of Gd-EOB-DTPA can predict reserved liver function.
\end{abstract}

Keywords: Liver functional reserves, model score for end-stage liver disease, signal intensity

Currently, many causes of liver dysfunction, including viral hepatitis, hepatic steatosis, cholestasis and chemotherapy-associated steato-hepatitis have been recognized. Chronic viral hepatitis is a major cause of cirrhosis and can lead to hepatocellular carcinoma (HCC) [1, 2]. Several tests and clinical scores for estimating liver functional reserve are well established before considering hepatic resection or liver transplantation, e.g. conventional liver function tests, Child-Turcotte-Pugh (CTP) score, the model for end-stage liver disease (MELD) score and indocyanine green test [1].

The CTP score has generally been used, but the MELD score has recently replaced the CTP score [3]. An increase in MELD score is associated with a decrease in residual liver function [4]. The MELD

Correspondence to: Nattaporn Tanpowpong, Department of Radiology, Faculty of Medicine, Chulalongkorn University, Bangkok 10330, Thailand. E-mail: tanpowpongn@gmail.com score was formulated as 3.78* $\log _{\mathrm{e}}($ bilirubin $[\mathrm{mg} / \mathrm{dL}])$ $+11.2 * \log _{\mathrm{e}}(\mathrm{INR})+9.57 * \log _{\mathrm{e}}($ creatinine $[\mathrm{mg} / \mathrm{dL}])+$ $6.43[5]$.

Gadolinium diethylenetriamine-pentaacetic acid (Gd-EOB-DTPA, gadoxetic acid) is a developed contrast agent with preferential uptake by the hepatocytes and has been used for almost two decades [6-8]. A rapid and specific hepatocyte uptake with biliary excretion is observed in approximately 50\% of the injected dose. The signal intensity of the liver gradually increases after injection of contrast agent with a peak liver signal intensity about 20 minutes followed by a plateau-like enhancement over 2 hours [6-8]. The amount of contrast uptake is related to liver functional reserve.

The aim of this study is to evaluate a correlation between liver signal intensity in the hepatobiliary phase of Gd-EOB-DTPA by using percentage enhancement and liver functional reserves using the MELD score. 


\section{Material and methods}

This study was approved by our local institutional review board. Informed consent was waived because there was neither any additional intervention nor pulse sequence on magnetic resonance (MR) imaging.

Data from all patients who underwent magnetic resonance (MR) imaging with Gd-EOB-DTPA from January 2006 to November 2011 were retrospectively collected. The patients who had serum creatinine higher than $1.5 \mathrm{mg} / \mathrm{dL}$ or without available parameters to estimate MELD score within 4 weeks of the MR imaging, were excluded from the study. The parameters were recorded as follows: INR, serum total bilirubin level and serum creatinine. After that, the MELD score was determined.

By using a 1.5 Tesla MR system (Signa EXCITE HD systems, GE Medical Healthcare with an 8channel phased array coil), the imaging sequences included a fat-suppressed three-dimensional fast spoiled-gradient recalled echo sequence (liver acceleration volume acquisition ([LAVA]) before and after injection of contrast material. Post-contrast T1weighted images were obtained at 20 minutes after administration of Gd-EOB-DTPA (repetition time, 4.2 $\mathrm{ms}$; echo time, $2.0 \mathrm{~ms}$; flip angle, $12^{\circ}$; slice thickness, $4.4 \mathrm{~mm}$; slice spacing, $2.2 \mathrm{~mm}$; acquisition, $320 \times 192$; number of excitations, 0.75 ; image matrix, $512 \times 512$ ). Each patient was injected the standard dose of GdEOB-DTPA (Primovist, Bayer Schering Pharma, Berlin, Germany) at a dose of 25 micromole $/ \mathrm{kg}$ of body weight at a flow rate of $2 \mathrm{~mL} / \mathrm{s}$ using a power injector, followed by a $20 \mathrm{~mL}$ saline flush at a rate of $2 \mathrm{~mL} / \mathrm{s}$.

Imaging analysis was performed by two boardcertificated radiologists broadly following the method used by Tajima et al. [10] as follows. A signal-to-noise ratio (SNR) in the liver parenchyma on LAVA images before and 20 minutes after contrast injection were measured on picture archiving and communication systems (PACS). Regions of interest (ROIs) drawn as round shapes at the hepatic segment II and VII in the Couinaud's liver segment classification. The ROIs were drawn on normal liver parenchyma away from a focal liver lesion or on an abnormal bile duct dilatation. The ROIs were also drawn at each corner of the background (4 ROIs in total) at the same level (Figure 1). ROIs before and after contrast injection were compared at the same regions in each patient. The signal intensity of liver and background were calculated as the mean signal intensity (SI liver and SI background, respectively). The area of each ROI was approximately $200 \mathrm{~mm}^{2}$. Liver SNR was calculated as follows; SNR = SI liver/SI background.

SNRs of liver parenchyma before and after contrast injection were used to measure a percentage enhancement. The percentage enhancement was calculated as follows:

Percentage enhancement $=$

$\frac{(\text { Postcontrast SNR }- \text { Precontrast SNR) } * 100}{\text { Precontrast SNR }}$

Pearson's correlation was computed between the percentage enhancement and the MELD score. Interobserver reliability of the percentage enhancement was determined by intraclass correlation coefficient (ICC). The scale for the ICC for interobserver reliability was categorized: less than $0.20=$ poor, $0.21-$ $0.40=$ fair, $0.41-0.60=$ moderate, $0.61-0.80=$ substantial, and 0.81-1.0 = near-perfect. Statistical analyses were computed with a statistical software package (SPSS, version 16.0.0; IBM Corporation, Armonk, NY). $P<0.05$ was considered statistically significant.
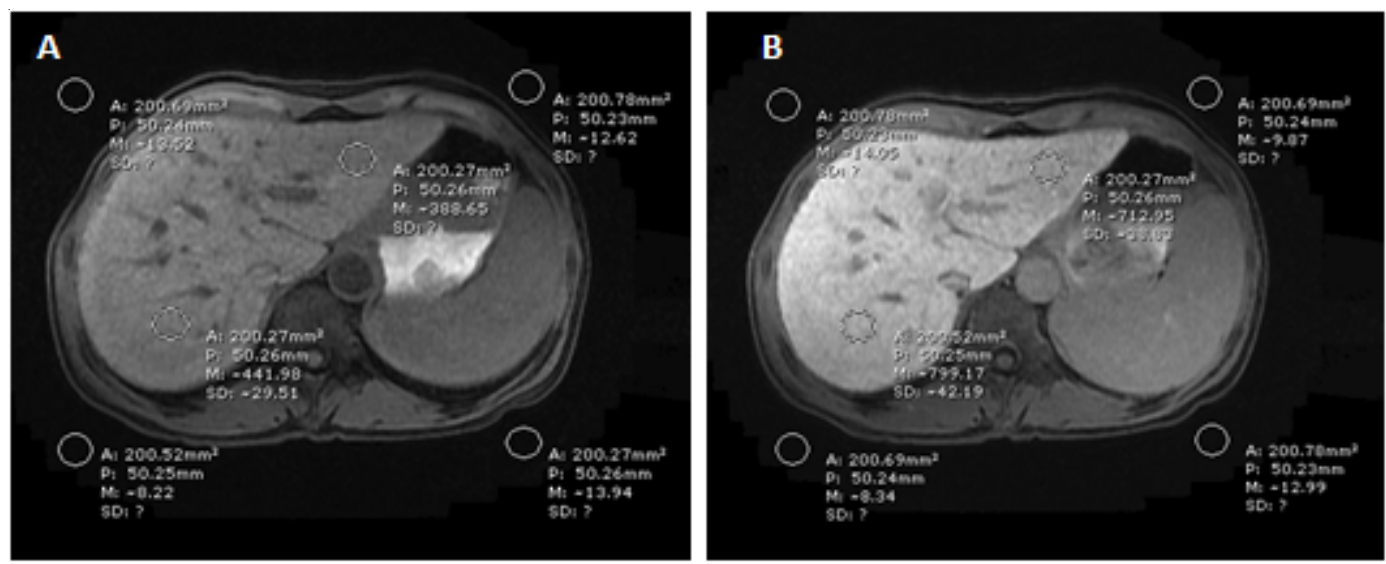

Figure 1. Positions of regions of interest (ROIs) drawn in the liver and background. A: Position of ROIs drawn in the liver and background on LAVA image before contrast injection. B: Position of ROIs drawn in the liver and background on LAVA image at 20 minutes after contrast injection. 


\section{Results}

Thirty-six patients were enrolled in the study: 26 were men and 10 were women. Their mean age was 58 years and the age range was between 38 and 76 years.

We found a negative correlation between the percentage enhancement and the MELD score
( $P<0.01, r=-0.545)$ (Figure 2 and Table 1). The SNR at 20 minutes after the Gd-EOB-DTPA injection also had a negative correlation with the MELD score $(P<0.01, r=-0.460)$ (Figure 3 and Table 1). There was substantial agreement of interobserver reliability of the percentage enhancement $($ ICC $=0.675)$

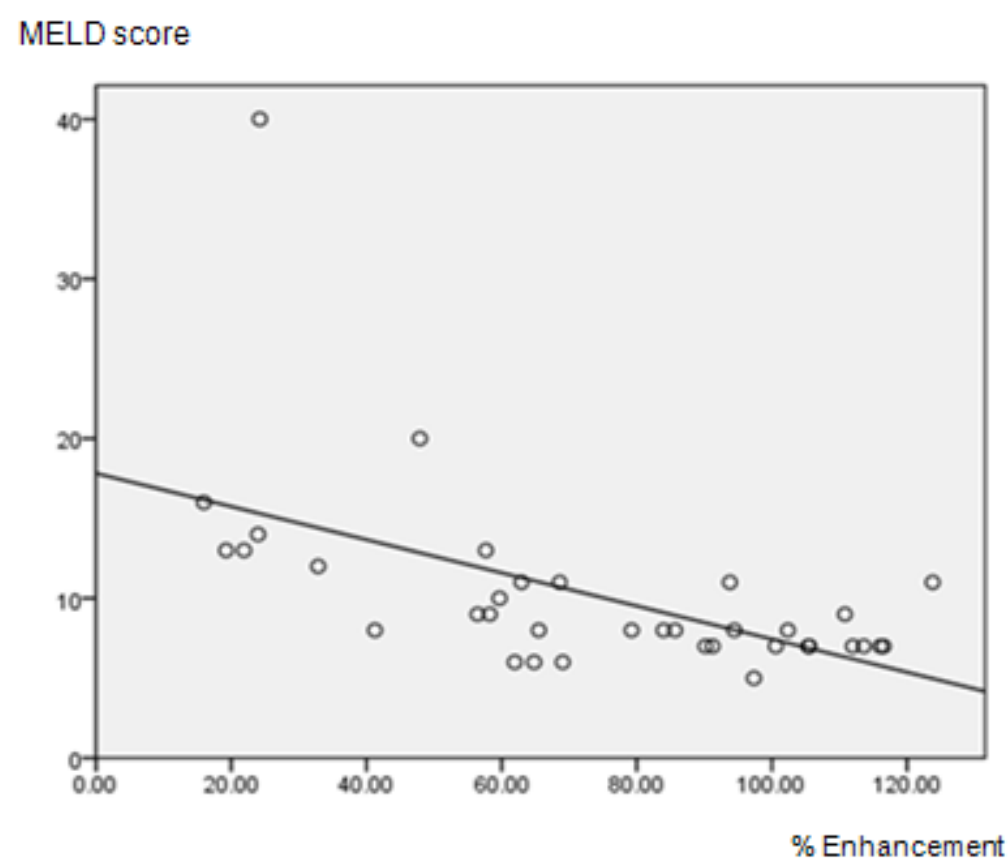

Figure 2. Scatter plot with regression line demonstrate correlation between percentage enhancement and the model for end-stage liver disease (MELD) score $(r=-0.545, P<0.01)$

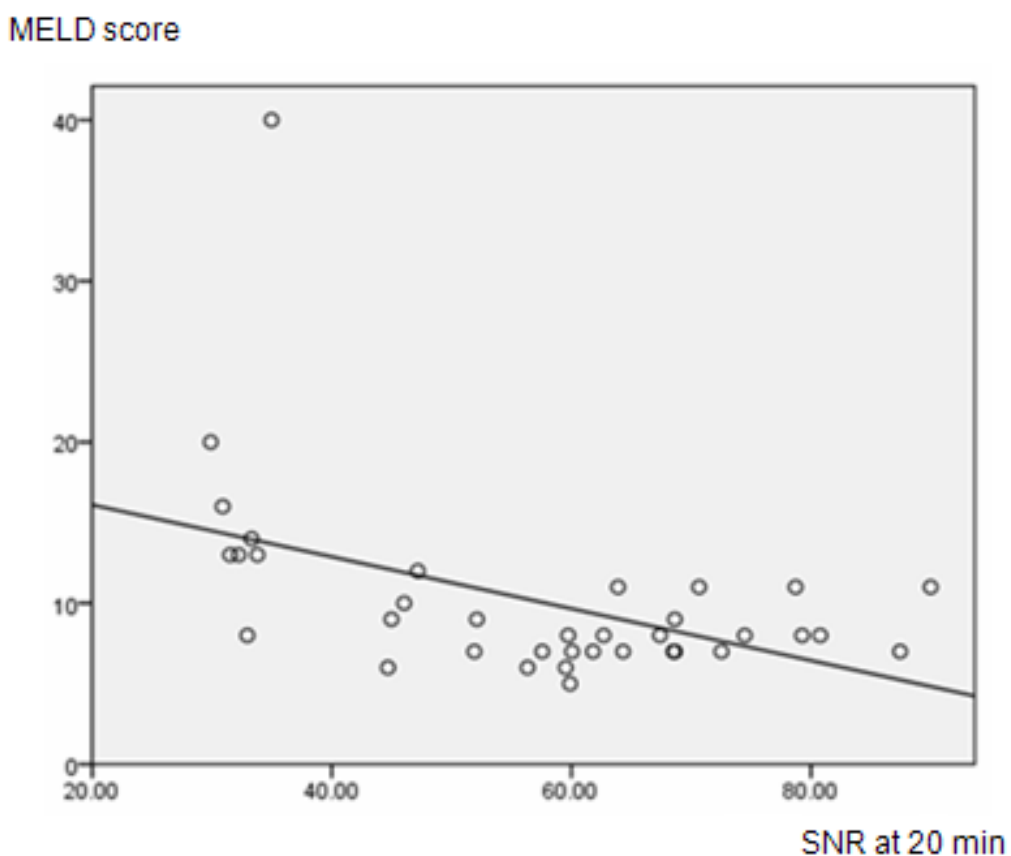

Figure 3. Scatter plot with regression line demonstrating the correlation between signal-to-noise ratio (SNR) at 20 minutes after gadolinium diethylenetriamine-pentaacetic acid injection and the model for end-stage liver disease (MELD) score $(r=-0.460, P<0.01)$. 
Table 1. Calculated Pearson correlation reveals significant correlation between the percentage enhancement, the signal-to-noise ratio (SNR) at 20 minutes after gadolinium diethylenetriamine-pentaacetic acid injection and the model for end-stage liver disease (MELD) score

\begin{tabular}{ll}
\hline & MELD score \\
\hline SNR at 20 minutes & \\
Pearson Correlation & -0.460 \\
$P$ (2-tailed) & 0.005 \\
N & 36 \\
Percentage enhancement & \\
Pearson Correlation & -0.545 \\
$P$ (2-tailed) & 0.001 \\
N & 36 \\
\hline
\end{tabular}

\section{Discussion}

To date, available studies assessing the relationship between reserve liver function and hepatic enhancement are limited. Ryeom et al. [9] studied the relationship in animals and did not use the SI on the MR imaging. In 2009, the relationship between reserve liver function and SI in hepatobiliary phase of Gd-EOB-DTPA-enhanced MR imaging in human was introduced by Tajima et al. [10]. The authors divided patients into two groups; chronic liver dysfunction and the normal liver function, and found that the degree of liver enhancement in the hepatobiliary phase may reflect liver function.

Katsube et al. [11, 12] suggested that evaluation of hepatic uptake of Gd-EOB-DTPA using T1 mapping and the $\mathrm{T} 2 *$ reduction rate, and the increase rate of the liver-to-muscle signal intensity ratio can help estimate liver function according to CTP score. Tamada et al. [13] concluded that hepatic parenchymal enhancement obtained using Gd-EOB-DTPA was affected by the severity of cirrhosis using CTP score. Nakamura et al. [14] revealed that the relative enhancement of the liver-to-phantom intensity ratio in the CTP $\mathrm{C}$ group was significantly lower than in the CTP A and B groups. Based on the pharmacokinetics of Gd-EOB-DTPA and some information from previous studies, we used the percentage enhancement to determine the liver function reserve with a newer model to determine residual liver function, the MELD score. We also evaluated the correlation between the SNR at 20 minutes after Gd-EOB-DTPA with the MELD score. We found that the lower the percentage enhancement and the SNR at hepatobiliary phase (20 minutes after Gd-EOB-DTPA injection), the higher the MELD score. This statistically significant correlation leads us to study further and obtain data, which can be used as a complement of, or a replacement for, the tests and scores for liver function reserve estimation.

The recent report by Verloh et al. [15] also focused on the usefulness of Gd-EOB-DTPAenhanced MRI to determine the hepatic functional reserve and the results are quite similar as ours; the uptake of Gd-EOB-DTPA in hepatocysts is strongly affected by liver function. In our study, we added on information about the interobserver reliability of the percentage enhancement as well, which shows substantial agreement. This means there is some difficulty for practical use and interpretation by this method.

There are some limitations of our study. Firstly, we can conclude only statistically significant correlation between the percentage enhancement and SNR at 20 minutes after Gd-EOB-DTPA injection with the liver function reserve. Further studies are required to quantitatively estimate the hepatic function reserves from the liver SI. Secondly, there were relatively small number of samples. Further investigation with a larger sample size should be conducted to ensure the significant correlation.

\section{Conclusion}

The percentage enhancement and liver SI in the hepatobiliary phase of Gd-EOB-DTPA is helpful in predicting liver functional reserves.

\section{Acknowledgment}

We would like to thank the staff of the Department of Radiology, Chulalongkorn University, for their support and advice. All authors declare no conflict of interest.

\section{References}

1. Seyama Y, Kokudo N. Assessment of liver function for safe hepatic resection. Hepatol Res. 2009; 39: 107-16.

2. Manizate F, Hiotis SP, Labow D, Roayaie S, Schwartz M. Liver functional reserve estimation: state of the art and relevance to local treatments. Oncology. 2010; 78: 131-4.

3. Malinchoc M, Kamath PS, Gordon FD, Peine CJ, Rank J, Borg PC. A model to predict poor survival in patients undergoing transjugular intrahepatic 
portosystemic shunts. Hepatology. 2000; 31:864-71.

4. Botta F, Giannini E, Romagnoli P, Fasoli A, Malfatti F, Chiarbonello B, et al. MELD scoring system is useful for predicting prognosis in patients with liver cirrhosis and is correlated with residual liver function: a European study. Gut. 2003; 52:134-9.

5. Kamath PS, Wiesner RH, Malinchoc M, Kremers W, Therneau TM, Kosberg CL, et al. A model to predict survival in patients with end-stage liver disease. Hepatology. 2001; 33:464-70.

6. Hamm B, Staks T, Muhler A, Bollow M, Taupitz M, Frenzel T, et al. Phase I clinical evaluation of GdEOB-DTPA as a hepatobiliary MR contrast agent: safety, pharmacokinetics, and MR imaging. Radiology. 1995; 195:785-92.

7. Reimer P, Rummeny EJ, Shamsi K, Balzer T, Daldrup HE, Tombach B, et al. Phase II clinical evaluation of Gd-EOB-DTPA: dose, safety aspects, and pulse sequence. Radiology. 1996; 199:177-83.

8. Bluemke DA, Sahani D, Amendola M, Balzer T, Breuer J, Brown JJ, et al. Efficacy and safety of MR imaging with liver-specific contrast agent: U.S. multicenter phase III study. Radiology. 2005; 237: 89-98.

9. Ryeom HK, Kim SH, Kim JY, Kim HJ, Lee JM, Chang YM, et al. Quantitative evaluation of liver function with MRI Using Gd-EOB-DTPA. Korean J Radiol. 2004; 5:231-9.

10. Tajima T, Takao H, Akai H, Kiryu S, Imamura H,
Watanabe Y, et al. Relationship between liver function and liver signal intensity in hepatobiliary phase of gadolinium ethoxybenzyl diethylenetriamine pentaacetic acid-enhanced magnetic resonance imaging. J Comput Assist Tomogr. 2010; 34:362-6.

11. Katsube T, Okada M, Kumano S, Hori M, Imaoka I, Ishii $\mathrm{K}$, et al. Estimation of liver function using T1 mapping on Gd-EOB-DTPA-enhanced magnetic resonance imaging. Invest Radiol. 2011; 46:277-83.

12. Katsube T, Okada M, Kumano S, Imaoka I, Kagawa Y, Hori M, et al. Estimation of liver function using T2* mapping on gadolinium ethoxybenzyl diethylenetriamine pentaacetic acid enhanced magnetic resonance imaging. Eur J Radiol. 2012; 81:1460-4.

13. Tamada T, Ito K, Higaki A, Yoshida K, Kanki A, Sato $\mathrm{T}$, et al. Gd-EOB-DTPA-enhanced MR imaging: evaluation of hepatic enhancement effects in normal and cirrhotic livers. Eur J Radiol. 2011; 80:e311-6.

14. Nakamura S, Awai K, Utsunomiya D, Namimoto T, Nakaura T, Morita K, et al. Chronological evaluation of liver enhancement in patients with chronic liver disease at Gd-EOB-DTPA-enhanced 3-T MR imaging: does liver function correlate with enhancement? Jpn J Radiol. 2012; 30:25-33.

15. Verloh N, Haimerl M, Zeman F, Schlabeck M, Wiggermann $\mathrm{P}$, et al. Assessing liver function by liver enhancement during the hepatobiliary phase with Gd-EOB-DTPA-enhanced MRI at 3 Tesla. Eur Radiol. 2014; 24:1013-9. 